\title{
Evolução da gravidez e resultados perinatais em transplantadas renais
}

\author{
Pregnancy and perinatal outcomes in women with renal transplantation
}

Leandro Gustavo de Oliveira ${ }^{1}$, Nelson Sass², Luiz Camano ${ }^{3}$, José Osmar Medina Pestana ${ }^{4}$

RESUMO

Objetivo: avaliar as inter-relações recíprocas entre o transplante renal e a gravidez por meio da análise das intercorrências clínicas e obstétricas, bem como da investigação do desfecho perinatal. Métodos: foi analisada série retrospectiva de 39 casos de gestação em 37 portadoras de transplante renal no período de janeiro de 1997 a dezembro de 2003. Foi criado um grupo controle composto por 66 grávidas sem doenças clínicas prévias, que freqüentaram pré-natal e deram à luz em $2002 \mathrm{e}$ 2003. Esse grupo foi utilizado para possíveis comparações para as variáveis pré-eclâmpsia, ruptura prematura de membranas, prematuridade e crescimento intra-uterino restrito. Como características demográficas dos dois grupos foram relatados média de idade no momento da concepção, raça e os antecedentes obstétricos. Quanto às características do transplante relatamos o tipo de doador e o esquema imunossupressor utilizado. As variáveis clínicas estudadas foram hipertensão arterial crônica, anemia e infecção do trato urinário. As variáveis relativas ao transplante foram o intervalo entre a cirurgia e a concepção, a ocorrência de disfunção, rejeição e perda do enxerto. Quanto às variáveis obstétricas, analisamos a via de parto, a incidência de pré-eclâmpsia e a ruptura prematura de membranas. Entre os resultados perinatais consideramos a incidência de prematuridade e crescimento intra-uterino restrito. Relacionamos estes resultados aos níveis de creatinina das transplantadas renais no início do pré-natal. Para avaliação estatística foram utilizados os testes de $\chi^{2}$ e exato de Fisher. Em todos os casos, o nível de rejeição para a hipótese de nulidade foi sempre fixado em valor menor ou igual a 0,05 (5\%). Resultados: a média de idade das pacientes no momento da concepção foi de 27 anos. $\mathrm{O}$ doador vivo foi o tipo mais freqüente. Entre as drogas imunossupressoras, a ciclosporina foi a mais utilizada. Hipertensão arterial crônica foi observada em $82 \%$ dos casos, anemia em 77\% e infecção do trato urinário em 38,5\%. A incidência de disfunção do enxerto foi de 47,4\%, tendo sido a pré-eclampsia a sua principal causa. Perda do transplante ocorreu em 10,2\% dos casos. Quanto à via de parto, a cesariana foi realizada em 53,8\%, tendo como principais indicações as síndromes hipertensivas. Pré-eclampsia ocorreu em $28,2 \%$ das transplantadas. Quanto aos resultados perinatais, prematuridade ocorreu em $46,1 \%$ dos casos, apresentando relação significativa com os níveis de creatinina maiores ou iguais a $1,5 \mathrm{mg} / \mathrm{dL}$ no primeiro trimestre. Outra intercorrência observada foi restrição de crescimento intra-uterino, verificada em $41,0 \%$ das gestações. A mesma não apresentou, entretanto, relação com os níveis séricos de creatinina. Conclusões: o grupo de estudo foi constituído por pacientes jovens. Hipertensão arterial crônica, anemia e infecção do trato urinário foram intercorrências clínicas bastante freqüentes. Disfunção do enxerto ocorreu em quase metade dos casos, devendo ser rastreada durante o pré-natal. Essas pacientes podem ainda evoluir para a perda do transplante, cujas causas podem ser obstétricas ou não. Quanto à via de parto, a cesariana teve incidência elevada, entretanto lembramos que a via de parto deve ser sempre de indicação obstétrica. Pré-eclampsia ocorreu de forma significativamente maior, devendo ser essas pacientes consideradas como condição de risco para essa complicação. Prematuridade e restrição de crescimento intra-uterino permanecem como importantes complicações perinatais, sendo que a prematuridade se relaciona com a função do transplante.

PALAVRAS-CHAVE: Transplante renal; Gravidez; Imunossupressão; Hipertensão; Pré-eclâmpsia

\section{ABSTRACT}

Purpose: to evaluate the relationship between renal transplantation and pregnancy through the analysis of clinical and obstetric intercurrent events and perinatal outcomes. Methods: a retrospective series of 39 cases of pregnancy in 37 women with renal transplantation from January 1997 to December 2003 was evaluated. A control group consisted of 66 pregnant women with no previous clinical pathologies. This group received prenatal care and these patients delivered during 2002

Universidade Federal de São Paulo - Escola Paulista de Medicina

1 Pós-graduando do Departamento de Obstetrícia - Universidade Federal de São Paulo - UNIFESP - São Paulo (SP) - Brasil.

2 Professor Adjunto do Departamento de Obstetrícia - Universidade Federal de São Paulo - UNIFESP - São Paulo (SP) - Brasil.

3 Professor Titular do Departamento de Obstetricia - Universidade Federal de São Paulo - UNIFESP - São Paulo (SP) - Brasil.

4 Prof. Adjunto da Disciplina de Nefrologia - Chefe da Unidade de Transplante Renal - Universidade Federal de São Paulo - Escola Paulista de Medicina Correspondência: Leandro Gustavo de Oliveira

R. Dr. Diogo de Faria, 1226; apto 113 - Vila Clementino - São Paulo - SP - Telefone: (11) 5549 - 9911 - Fax: (11) 3815 - 6308 - e-mail: leminas@ig.com.br Recebido em: 8/4/2005 Aceito com modificaçōes em: 13/6/2005

Rev Bras Ginecol Obstet. 2005;27(6):316-22 
and 2003. Preeclampsia, premature rupture of membranes, premature delivery, and intrauterine growth restriction were used to compare these variables. Demographic characteristics of these groups were related to the mean age at conception, ethnic characteristics and obstetric past. Regarding renal transplantation the type of donator and used immunosuppressive drugs were evaluated. The studied clinical variables were chronic hypertension, anemia and urinary tract infection. The interval between the surgery and conception, occurrence of dysfunction, rejection and loss of the allograft were characteristcs related to the allograft. Obstetric variables were related to the type of delivery, incidence of preeclampsia and premature rupture of membranes. Perinatal outcomes were premature delivery and intrauterine growth restriction and these results were compared with renal function. The used statistical methods were the $\chi^{2}$ and Fisher's exact tests. The significance level was fixed always as less than or equal to $0.05(5 \%)$. Results: the mean age at conception was 27 years. The live donator was the most frequent among the patients. Among the immunosuppressive drugs, cyclosporine was the most used. Chronic hypertension occurred in $82 \%$ of the cases, anemia in $77 \%$ and urinary tract infection in $38.5 \%$. The incidence of renal dysfunction was $47.4 \%$ and preeclampsia was the main cause. The loss of the renal transplantation occurred in $10.2 \%$. Delivery by cesarean section was performed in $53.8 \%$ of the patients, and the main causes were hypertensive syndromes. Preeclampsia occurred in $28.2 \%$. Among the perinatal outcomes, premature delivery occurred in $46.1 \%$ of the cases, with a significant relation to creatinine level greater than or equal to $1.5 \mathrm{mg} / \mathrm{dL}$ at the start of prenatal care. Another observed intercurrent event was intrauterine growth restriction, which occurred in $41.0 \%$, and here we found no relation between this event and creatinine levels. Conclusions: young patients constituted the study group. Chronic hypertension, anemia and urinary tract infection were very common. Renal dysfunction was frequent and must be investigated during prenatal care. There were four cases of loss of the transplant due to clinical or obstetric causes. Cesarean delivery had the highest incidence, but vaginal delivery should be the first choice in these cases. Preeclampsia occurred very frequently and this complication should be considered as a high risk. Preterm delivery and intrauterine growth restriction were the main perinatal complications. Premature deliveries before 37 weeks of gestation were related to allograft function.

KEYWORDS: Kidney transplantation; Pregnancy; Immunosuppression; Hypertension; Pre-eclampsia

\section{Introdução}

Conforme os dados da Associação Brasileira de Transplante de Órgãos (ABTO), o número de transplantes renais (TR) no Brasil tem-se elevado constantemente ${ }^{1}$, tanto devido à criação de programas de captação de órgãos com o auxílio governamental como, também, ao aumento do número de centros transplantadores. Há, segundo essa associação, 153 centros de TR cadastrados no país ${ }^{1}$ e de acordo com Medina-Pestana et al. ${ }^{2}$ existem no Brasil cerca de 45000 pacientes em diálise, que representam importante demanda para esses centros.

No ano de 2003 o Hospital do Rim e Hipertensão da Universidade Federal de São Paulo foi responsável pela realização de 527 transplantes renais, sendo considerado atualmente o serviço que mais realiza o procedimento no mundo. Sabemos que cerca de $7 \%$ desses pacientes são representados por mulheres em idade fértil, que conservam a capacidade reprodutiva.

No que diz respeito às inter-relações entre doença renal e a possibilidade de gravidez, verifica-se que mulheres portadoras de insuficiência renal crônica apresentam freqüentemente infertilidade ${ }^{3}$. Estima-se uma taxa de gestação de 1:200 entre aquelas submetidas ao tratamento dialítico ${ }^{4,5}$. Por outro lado, após o transplante observa-se notável elevação das possibilidades de con- cepção, com relatos de gravidez na literatura internacional de 1 para $50 \operatorname{casos}^{4,5}$.

Em 1958, foi relatada a primeira gestação em portadora de TR de 22 anos de idade, que havia recebido o enxerto de sua irmã gêmea idênti$\mathrm{ca}^{6}$. A partir daí passaram a ser freqüentes os relatos a esse respeito ${ }^{7}$.

Diante da ampliação das possibilidades reprodutivas observadas após o TR, deparamo-nos com uma condição na qual a interação da gestação com as características clinicas próprias da paciente transplantada e o uso de drogas imunossupressoras exigem seguimento diferenciado durante o ciclo gravídico-puerperal.

Temos observado crescente número de gestantes portadoras de transplante renal e por isso fomos motivados a registrar as importantes interrelações reciprocas verificadas nesses casos. Ademais, estamos certos de que os progressos observados nessa área impõem a interação de várias especialidades, incluindo a Obstetrícia, com o intuito de proporcionar melhor acompanhamento de portadoras de transplantes e dessa forma podermos contribuir para o incremento de sua qualidade de vida.

Neste estudo relatamos as intercorrências clínicas, obstétricas e os resultados perinatais encontrados em 39 gestações em 37 portadoras de transplante renal. 
Métodos

Esse foi um estudo retrospectivo realizado na Universidade Federal de São Paulo - Escola Paulista de Medicina. Foram incluídas 39 gestações em 37 portadoras de transplante renal que deram à luz entre janeiro de 1997 e dezembro de 2003. Como critérios de inclusão admitimos as pacientes que realizaram todo o seguimento pré-natal e deram à luz no Hospital São Paulo. Foram excluidos os casos que terminaram em abortamento e as gestações gemelares. Foi estabelecido um grupo controle constituído por 66 pacientes com gestação única que iniciaram o pré-natal sem intercorrências obstétricas e sem doenças clínicas prévias. Todas essas pacientes também deram à luz no Hospital São Paulo entre janeiro de 2002 e dezembro de 2003 e apresentaram ou não intercorrências durante o pré-natal. O grupo controle foi utilizado para comparação apenas de algumas variáveis que serão descritas a seguir (pré-eclâmpsia, ruptura prematura de membranas, prematuridade e crescimento intra-uterino restrito).

Como características demográficas dos dois grupos foram relatados a média de idade no momento da concepção, a raça e os antecedentes obstétricos. Quanto às características do transplante relatamos o tipo de doador e o esquema imunossupressor utilizado. As variáveis estudadas foram divididas em clinicas, relativas ao transplante, obstétricas e resultados perinatais. As variáveis clinicas foram hipertensão arterial crônica, anemia e infecção do trato urinário. As variáveis relativas ao transplante foram o intervalo entre a cirurgia e a concepção, a ocorrência de disfunção, rejeição e perda do enxerto. Quanto às variáveis obstétricas analisamos a via de parto, a incidência de préeclâmpsia e a ruptura prematura de membranas. Entre os resultados perinatais consideramos a incidência de prematuridade e o crescimento intrauterino restrito (CIUR). Procuramos relacionar estes dois resultados aos niveis de creatinina das transplantadas renais no início do pré-natal.

Quanto às características demográficas dos grupos de estudo e controle verificamos que a faixa etária variou de 19 a 36 anos, com média de 27 anos. A distribuição da raça foi semelhante nos dois grupos comparados e quanto aos antecedentes obstétricos, houve distribuição homogênea entre nuliparas, primiparas e multíparas. Quanto ao tipo de doador, 26 pacientes haviam recebido o órgão de doador vivo, ao passo que 13 de doador cadáver. No que diz respeito ao esquema imunossupressor (Tabela 1), a maioria das pacientes utilizavam ciclosporina.
Tabela 1 - Esquema imunossupressor utilizado durante as 39 gestações em portadoras de transplante renal.

\begin{tabular}{lrr}
\hline Imunossupressores & $\mathbf{n}$ & \% \\
\hline Ciclosporina + azatioprina + prednisona & 21 & 53,8 \\
Ciclosporina + azatioprina & 4 & 10,3 \\
Ciclosporina + prednisona & 6 & 15,4 \\
Ciclosporina & 2 & 5,1 \\
Azatioprina + prednisona & 2 & 5,1 \\
Tacrolimus + azatioprina + prednisona & 4 & 10,3 \\
Total & 39 & 100,0 \\
\hline
\end{tabular}

n: número.

Quanto às variáveis clínicas definimos como hipertensão arterial crônica a pressão arterial sistólica maior ou igual a $140 \mathrm{mmHg}$ e/ou pressão arterial diastólica maior ou igual a $90 \mathrm{mmHg}$, diagnosticadas previamente à gestação ou antes da $20^{\mathrm{a}}$ semana de idade gestacional. Definimos como anemia a presença de concentrações de hemoglobina sangüínea inferiores a $11,0 \mathrm{~g} / \mathrm{dL}$ em qualquer período da gestação, parto ou puerpério. Infecção do trato urinário foi diagnosticada por urocultura, cuja contagem de colônias foi superior a 100.000 colônias $/ \mathrm{mL}$ de urina.

No que se refere às variáveis relacionadas ao TR, a disfunção do enxerto foi considerada pela elevação dos níveis plasmáticos de creatinina além de 0,3 mg/dL em qualquer período da gestação. Em face das situações em que se observou disfunção do enxerto, a rejeição ao transplante foi diagnosticada pelo padrão histológico verificado a partir de biópsia renal e/ou diante de melhora da função do órgão após a administração de pulsoterapia com corticosteróides e/ou imunossupressores. Para tanto foi considerado qualquer período da gestação, parto ou puerpério. Perda do transplante foi caracterizada quando houve perda total da função renal, acarretando o retorno ao tratamento dialítico, em qualquer período da gestação, parto ou puerpério.

Quanto às variáveis obstétricas definimos como pré-eclâmpsia a pressão arterial sistólica maior ou igual a $140 \mathrm{mmHg}$ e/ou pressão arterial diastólica maior ou igual a $90 \mathrm{mmHg}$, diagnosticadas pela primeira vez após a $20^{\mathrm{a}}$ semana de idade gestacional, associada à presença de proteinúria igual ou superior a $0,3 \mathrm{~g}$ em urina de 24 horas em pacientes que previamente não apresentavam proteinúria. Naquelas pacientes que tinham proteinúria desde o início da gestação foi considerada significativa elevação três vezes superior aos valores iniciais. Pré-eclâmpsia sobreposta foi considerada a piora dos niveis pressóricos (elevação igual ou superior a $30 \mathrm{mmHg}$ na pressão arterial sistólica e/ou igual ou superi- 
or a $15 \mathrm{mmHg}$ na pressão arterial diastólica) associada a proteinúria, já descrita anteriormente. O diagnóstico de ruptura prematura das membranas ovulares foi feito quando a mesma ocorreu antes do início do trabalho de parto e após a $22^{\mathrm{a}}$ semana de idade gestacional.

Entre os resultados perinatais foram considerados como pré-termo os recém-nascidos com idade gestacional maior do que 22 semanas e menor do que 37 semanas completas. Já o CIUR foi diagnosticado naqueles recém-nascidos que se encontraram abaixo do percentil 10 para a idade gestacional de acordo com curva de crescimento utilizada pelo Departamento de Obstetrícia e Disciplina de Pediatria Neonatal da UNIFESP $\mathrm{EPM}^{8}$.

A análise da associação entre as variáveis obstétricas e os resultados perinatais foi realizada utilizando-se os testes $\chi^{2}$ e exato de Fisher, quando adequados. Adotou-se o nivel de significância 0,05 (5\%). Algumas variáveis foram apenas apresentadas sob a forma de percentagem.

Esse trabalho foi aprovado pelo Comitê de Ética em Pesquisa da UNIFESP-EPM.

\section{Resultados}

Quanto às intercorrências clínicas, 32 pacientes $(82,0 \%)$ apresentavaram hipertensão arterial crônica; $30(77,0 \%)$ apresentaram anemia e $15(38,5 \%)$ tiveram pelo menos um episódio de infecção do trato urinário durante a gestação.

Entre as variáveis relacionadas ao transplante, o intervalo entre a cirurgia e o momento da concepção foi menor do que dois anos em 12 $(30,8 \%)$ pacientes e maior do que dois anos em 27 $(69,2 \%)$, sendo o intervalo médio de 3,2 anos. Disfunção do enxerto (Tabela 2) ocorreu em 19 casos $(48,7 \%)$, duas pacientes apresentaram rejeição $(5,1 \%)$ e quatro $(10,2 \%)$ evoluíram para perda do transplante.

Tabela 2 - Causas de disfunção do enxerto em 39 gestações em portadoras de transplante renal.

\begin{tabular}{lrr}
\hline Causas & n & \% \\
\hline Não definida & 6 & 31,5 \\
Pré-eclâmpsia & 9 & 47,4 \\
Rejeição & 2 & 10,5 \\
Infecção do trato urinário & 1 & 5,3 \\
Recorrência de glomeruloesclerose segmentar focal & 1 & 5,3 \\
Total & 19 & 100,0 \\
\hline
\end{tabular}

n: número.
Quanto às causas de perda do enxerto, estas se deram em dois casos devido à rejeição, um caso devido à recorrência de glomeruloesclerose segmentar focal e um deles se relacionou a uma causa obstétrica, que foi choque hemorrágico seguido de choque séptico após parto domiciliar.

Parto cesáreo foi realizado em 21 (53,8\%) casos entre as portadoras de TR e suas principais indicações se relacionaram às síndromes hipertensivas. No que se refere às variáveis obstétricas (Tabela 3), pré-eclâmpsia foi diagnosticada em $11(28,2 \%)$ pacientes do grupo de estudo e em seis $(9,0 \%)$ do grupo controle, sendo essa diferença estatisticamente significante $\left(\chi^{2}\right.$ calculado (Yates) $=5,267 ; p=0,022)$. Ruptura prematura de membranas ocorreu em quatro $(10,3 \%)$ transplantadas e em duas $(3,0 \%)$ pacientes do grupo controle, não havendo diferença estatística nesse caso (teste exato de Fisher; $p=0,135$ ).

Tabela 3 - Intercorrências obstétricas e resultados perinatais em 39 gestações em portadoras de transplante renal co relação ao grupo controle.

\begin{tabular}{lcccc}
\hline Intercorrência & Grupo de estudo & \multicolumn{2}{c}{ Grupo controle } \\
& $\mathbf{n}$ & $\%$ & $\mathbf{n}$ & $\%$ \\
\hline Pré-eclâmpsia* $^{*}$ & 11 & 28,2 & 6 & 9,0 \\
RPM & 4 & 10,2 & 2 & 3,0 \\
Prematuridade* $^{*}$ & 18 & 46,1 & 9 & 13,6 \\
ClUR $^{*}$ & 16 & 41,0 & 12 & 18,1 \\
\hline
\end{tabular}

n: número; RPM: ruptura prematura de membranas; CIUR: crescimento intra-uterino restrito; ${ }^{*} p<0,005$.

Quanto aos resultados perinatais, $18(46,1 \%)$ recém-nascidos foram considerados pré-termo no grupo de estudo e nove $(13,6 \%)$ no grupo controle, sendo essa diferença estatisticamente significante $\left(\chi^{2}\right.$ calculado (Yates) $\left.=11,921 ; p=0,001\right)$. CIUR ocorreu em 16 fetos $(41,1 \%)$ do grupo de transplantadas e em $12(18,1 \%)$ do grupo controle e também foi considerado estatisticamente significante $\left(\chi^{2}\right.$ calculado (Yates) $=5,426 ; p=0,020)$.

Quando relacionamos prematuridade com os niveis de creatinina apresentados pelas transplantadas renais no início do pré-natal (Tabela 4) observamos que seis $(27,3 \%)$ das 22 pacientes com creatinina menor do que $1,5 \mathrm{mg} / \mathrm{dL}$ tiveram recém-nascidos pré-termo, ao passo que as 17 gestantes que iniciaram o pré-natal com niveis maiores ou iguais a $1,5 \mathrm{mg} / \mathrm{dL}$ tiveram $12(70,6 \%)$ prematuros. Esta diferença foi significante do ponto de vista estatístico $\left(\chi^{2}\right.$ calculado $\left.=7,240 ; p=0,007\right)$.

Analisando a incidência de CIUR e os níveis de creatinina no início do pré-natal (Tabela 4), ocorreram oito casos entre as pacientes com níveis de creatinina menores do que $1,5 \mathrm{mg} / \mathrm{dL}$ e oito entre aquelas com creatinina maior ou igual a $1,5 \mathrm{mg} / \mathrm{dL}$, não apresentando, portanto, diferença estatística $\left(\chi^{2}\right.$ calculado $\left.=0,453 ; p=0,501\right)$. 
Tabela 4 - Freqüência de prematuridade e crescimento intra-uterino restrito entre as 39 gestações em portadoras de transplante renal com relação ao nível de creatinina no $1^{\circ}$ trimestre.

\begin{tabular}{|c|c|c|c|c|c|c|c|c|c|c|c|c|}
\hline \multirow[t]{3}{*}{ Creatinina } & \multicolumn{6}{|c|}{ Prematuridade } & \multicolumn{6}{|c|}{ Crescimento intra-uterino restrito } \\
\hline & \multicolumn{2}{|c|}{ Sim } & \multicolumn{2}{|c|}{ Não } & \multicolumn{2}{|c|}{ Total } & \multicolumn{2}{|c|}{ Sim } & \multicolumn{2}{|c|}{ Não } & \multicolumn{2}{|c|}{ Total } \\
\hline & $\mathrm{n}$ & $\%$ & $\mathrm{n}$ & $\%$ & $\mathrm{n}$ & $\%$ & $\mathrm{n}$ & $\%$ & $\mathrm{n}$ & $\%$ & $\mathrm{n}$ & $\%$ \\
\hline$<1,5 \mathrm{mg} / \mathrm{dL}$ & 6 & 27,3 & 16 & 72,7 & 22 & 100,0 & 8 & 36,4 & 14 & 63,6 & 22 & 100,0 \\
\hline$\geq 1,5 \mathrm{mg} / \mathrm{dL}$ & 12 & 70,6 & 5 & 29,4 & 17 & 100,0 & 8 N.S. & 47,0 & 9 & 53,0 & 17 & 100,0 \\
\hline Total & 18 & & 21 & & 39 & & 16 & & 23 & & 39 & \\
\hline
\end{tabular}

n: número; * ${ }^{\circ}=0,007$ (significante); N.S. = 0,501 (não significante).

\section{Discussão}

De acordo com nossos resultados a média de idade das portadoras de transplante renal no momento da concepção foi de 27 anos, variando de 17 até 36 anos. Na literatura mundial, a média de idade relatada varia de $23 \operatorname{anos}^{9}$ até 29 anos ${ }^{10}$. O intervalo médio entre a cirurgia e o momento da concepção entre as pacientes do estudo foi de 3,2 anos. Esse resultado foi compativel com aqueles encontrados na literatura, que estão em torno de quatro anos ${ }^{5,9}$. Isso é importante, pois diversos autores insistem em considerar que o período mínimo para engravidar deve ser de dois anos após o procedimento ${ }^{4,11}$, permitindo assim o adequado controle imunossupressor e de doenças crônicas associadas, como a hipertensão arterial, o diabetes e o lúpus eritematoso sistêmico, entre outras. Ainda assim, importante parcela das nossas pacientes $(30,8 \%)$ engravidou antes de dois anos, demonstrando falta de planejamento por parte do casal. Isso torna imprescindivel orientação contraceptiva precoce, pois o retorno à fertilidade pode ser imprevisivel nesses casos, mesmo após longo período dialítico pregresso. O doador vivo continua sendo o mais freqüente nos casos de transplante renal, apesar da intensificação das campanhas de doação de órgãos e dos programas de captação. É importante frisar, todavia, que o doador ideal para os transplantes é o cadáver, pois evita a exposição do paciente hígido às possiveis complicações, tanto cirúrgicas quanto aquelas relacionadas à vida com um único rim².

Quanto às intercorrências clínicas observadas em gestantes portadoras de transplante renal, a hipertensão arterial crônica esteve presente em 32 delas $(82,0 \%)$, concordando com os relatos da literatura $^{12,13}$. Apesar de bastante prevalente, observamos que durante o acompanhamento prénatal a maioria das pacientes apresentou hipertensão de fácil controle, respondendo bem ao tratamento com um único hipotensor. Esse aspecto em relação ao manejo clínico da hipertensão arterial crônica nessas pacientes já havia sido re- latado por Davison ${ }^{11}$, em 1991 e, segundo esse autor, relaciona-se ao tempo de transplante.

A anemia é bastante freqüente em nefropatas crônicas, decorrente da baixa produção de eritropoetina. O mesmo acontece com as portadoras de transplante renal, que podem apresentar-se anêmicas, mesmo com niveis de creatinina dentro dos limites da normalidade. Considerando a hemodiluição fisiológica vigente na gestação, espera-se que possam ocorrer agravos durante esse periodo. Em nosso estudo, a anemia foi encontrada em 30 gestantes $(77,0 \%)$, concordante com os dados da literatura ${ }^{12-16}$. Durante o pré-natal, todas as pacientes receberam suplementação com ácido fólico e sulfato ferroso. Uma delas, por apresentar hemoglobina sangüinea igual a 7,8 $\mathrm{mg} / \mathrm{dL}$, recebeu transfusão de duas unidades de glóbulos vermelhos previamente a ser submetida ao parto cesáreo. Entre as puérperas, sete necessitaram de transfusão sanguínea durante os dois primeiros dias. Ao nosso ver, a suplementação vitaminica não é suficiente para restabelecer os niveis de hemoglobina durante a gestação de portadoras de TR, havendo como alternativa para o tratamento, a utilização de eritropoetina humana recombinante, conforme relatado por Al Shohaib ${ }^{17}$, em 1999.

As caracteristicas especiais apresentadas pelas transplantadas fazem da infecção do trato urinário uma condição temerária, levando à necessidade de profilaxia constante. Entre nossos casos, encontramos 15 pacientes com esse diagnóstico $(38,5 \%)$, semelhante aos dados da literatura $^{13,14}$. Diante desses valores, orientamos a utilização de antibioticoprofilaxia e independentemente disso a realização de urocultura mensal, visando identificar a bacteriúria assintomática. Em nossa vivência clínica, o tratamento foi instituído e orientado de acordo com o antibiograma e obtivemos boas respostas.

Quando avaliamos as anormalidades funcionais do enxerto ocorridas durante as 39 gestações, vimos que $48,7 \%$ delas apresentaram algum grau de disfunção. Na literatura mundial, encontramos relatos de que realmente ocorre prejuízo da função 
renal durante o ciclo gravídico-puerperal. Contudo, esse não exerce influência negativa na sobrevida do transplante ou da própria paciente ${ }^{12,18-20}$. Quanto à causa de detrimento da função renal, observamos que pré-eclampsia foi a principal, correspondendo a $47,4 \%$ dos casos. Ressaltamos que a avaliação funcional do enxerto deve ser feita rotineiramente mesmo em pacientes assintomáticas e sempre de forma multidisciplinar, pois outras causas como rejeição, recorrência de doenças prévias, obstruções ureterais e infecção do trato urinário podem ser responsáveis por seu detrimento. Outra questão importante é que em grande parte das vezes não se pode chegar a uma causa precisa para a disfunção, o que sugere que o próprio aumento volêmico observado na gestação e a sobrecarga que este representa para o rim transplantado podem ser causas disfuncionais, o que ao nosso ver pode justificar a indução de trabalho de parto assim que atingido o termo da gravidez.

Chamamos atenção para o fato de que uma das causas de perda do enxerto observada neste trabalho foi obstétrica e ocorreu devido a choque hemorrágico após parto domiciliar. Lembramos com esse caso que o rim transplantado é um órgão denervado e que por isso responde de maneira extremamente deficiente a variações dos níveis pressóricos ${ }^{21}$. Assim, episódios de hipotensão ocasionados por sangramentos excessivos ou procedimentos anestésicos podem mais facilmente conduzir à necrose tubular aguda, necrose cortical e perda do enxerto.

Entre as intercorrências obstétricas estudadas, a pré-eclâmpsia foi a principal, pois incidiu em 11 pacientes $(28,2 \%)$, valor semelhante aos referidos na literatura ${ }^{11-14}$. Essa incidência mostrou-se significativamente diferente das pacientes do grupo controle. Sabemos que diversas situações predispõem à ocorrência de pré-eclâmpsia, como diabetes, vasculopatias, nefropatias, etc. ${ }^{22}$. Entre algumas transplantadas, várias destas condições podem ser claramente definidas. Porém, em diversos casos não se pode identificar nem mesmo o motivo específico que levou ao transplante. Sendo assim, e por ser a pré-eclâmpsia uma causa de elevada morbidade materna e perinatal, acreditamos que estas pacientes devem ser consideradas de forma geral como mais suscetiveis a essa doença. Orientamos a partir disso que a préeclâmpsia deve ser rastreada exaustivamente nas portadoras de transplante renal, uma vez que seu diagnóstico é mais dificil nessas pacientes, já que a maioria delas possui hipertensão arterial crônica e freqüentemente apresentam proteinúria. Outra intercorrência bastante realçada pela literatura é a incidência de ruptura prematura de membranas, relatada em 27,6 e $20,0 \%{ }^{13,16}$. Em nosso estudo, tal intercorrência ocorreu em quatro casos $(10,2 \%)$, incidência esta que, ao compararmos com o grupo controle, não se apresentou estatisticamente significante. Acreditamos que essa redução relaciona-se com o acompanhamento prénatal pormenorizado no que diz respeito à investigação e tratamento das vaginoses e vulvovaginites, bastante freqüentes entre pacientes transplantadas devido ao estado imunológico. Quanto à via de parto, a cesariana foi realizada em $53,8 \%$ dos casos e suas principais indicações se relacionaram às sindromes hipertensivas. Quando avaliamos os dados da literatura, esses também apontam para as sindromes hipertensivas como sendo a principal indicação de partos cesáreos nas portadoras de $\mathrm{TR}^{12,14,23}$. Não observamos causas relacionadas à obstrução do canal de parto devido à presença do órgão transplantado, que se aloja na cavidade pélvica, sendo que de forma geral a via de parto foi determinada por indicação obstétrica, como já relatado por Davison ${ }^{11}$, em 1991.

Em nossa óptica, o parto transpélvico é sempre o preferido, pois se relaciona a menores perdas sangüíneas, menor risco infeccioso e menor possibilidade de trauma relacionado à abertura da cavidade abdominal e à histerotomia, que podem predispor a complicações importantes devido à proximidade do rim transplantado e de suas anastomoses, tanto as vasculares quanto a ureteral. Consideramos ainda infundada a chance de obstrução do canal de parto pela presença do enxerto na fossa ilíaca.

No que diz respeito aos resultados perinatais, tanto prematuridade quanto CIUR foram intercorrências de elevada importância e estatisticamente mais freqüentes do que no grupo controle, visto que 18 recém-nascidos $(46,1 \%)$ foram considerados pré-termo e $16(41,0 \%)$ apresentaram CIUR. Ambos os resultados foram concordantes com a literatura ${ }^{11-13,24}$.

Quando nos reportamos à função renal e aos resultados perinatais, sabemos que são comuns os trabalhos relacionando insuficiência renal crônica moderada (creatinina entre 1,5 e 2,4 mg/dL) ou grave (creatinina $\geq 2,5 \mathrm{mg} / \mathrm{dL}$ ) à prematuridade, com incidências de 59 e $100 \%{ }^{25,26}$, respectivamente. Sendo assim, imaginamos que as portadoras de TR que apresentam niveis de creatinina superiores ou iguais a $1,5 \mathrm{mg} / \mathrm{dL}$ possam também apresentar maior incidência dessa intercorrência. Em nosso trabalho encontramos incidência significativamente maior de prematuros entre aquelas pacientes que iniciaram o pré-natal com niveis de creatinina superiores ou iguais a $1,5 \mathrm{mg} / \mathrm{dL}$. Porém, quando comparamos essa variável com 
relação aos 16 casos (41,0\%) de CIUR, não observamos diferença estatística. Acreditamos, entretanto, que tais desfechos devem ser analisados com cautela, pois podem estar relacionados a um conjunto de fatores presentes nesses casos, como doenças pré-existentes e hipertensão arterial crônica, responsáveis habituais por insuficiência placentária e prematuridade eletiva.

Ao concluir, gostariamos de dizer, ao contrário do que muitos imaginam, que é perfeitamente aceitável que a maioria das portadoras de transplante renal possa engravidar. Consideramos apenas que a concepção deva ocorrer de forma planejada, em momentos nos quais as intercorrências clínicas e/ou relacionadas ao transplante estejam compensadas, e que, diante da gravidez, deve-se instituir assistência pré-natal diferenciada e multiprofissional.

\section{Referências}

1. Associação Brasileira de Transplante de Órgãos [homepage da Internet]. Informações sobre o transplante de órgãos no Brasil. São Paulo; 2004 [citado 2005 Fev 8]. Disponivel em: http://www.abto.com.br/

2. Medina-Pestana JO, Vaz ML, Park SI. Organ transplant in Brazil. Transplant Proc. 2002;34(2):441-3.

3. Basaran O, Emiroglu R, Secme S, Moray G, Haberal M. Pregnancy and renal transplantation. Transplant Proc. 2004;36 (1):122-4.

4. Sturgiss SN, Davison JM. Effect of pregnancy on long-term function of renal allografts. Am J Kidney Dis. 1992;19(2):167-72.

5. Lindheimer MD, Katz AI. Pregnancy in the renal transplant patient. Am J Kidney Dis. 1992;19(2):173-6.

6. Murray JE, Reid DE, Harrison JH, Merrill JP. Successful pregnancies after human renal transplantation. N Engl J Med. 1963;269:341-3.

7. Mezza E, Ogge G, Attini R, Rossetti M, Soragna G, Consiglio V, et al. Pregnancy after kidney transplantation: an evidence-based approach. Transplant Proc. 2004;36(10):2988-90.

8. Alexander GR, Himes JH, Kaufman RB, Mor J, Kogan M. A United States national reference for fetal growth. Obstet Gynecol. 1996;87(2):163-8.

9. Penn I, Makowski EL, Harris P. Parenthood following renal and hepatic transplantation. Transplantation. 1980;30(6):397-400.

10. Armenti VT, Ahlswede KM, Ahlswede BA, Jarrell BE, Moritz MJ, Burke JF. National transplantation pregnancy registry: outcomes of 154 pregnancies in cyclosporine-treated female kidney transplant recipients. Transplantation. 1994;57(4):502-6.
11.Davison JM. Dialysis, transplantation and pregnancy. Am J Kidney Dis. 1991;17(2):127-32.

12. Thompsom BC, Kingdon EJ, Tuck SM, Fernando ON, Sweny P. Pregnancy in renal transplant recipients: the Royal Free Hospital experience. QJM. 2003;96(11):837-44.

13. Bakr MA, el Said Ghaneim M, Fouda MA, Sally S, Moustafa FE, Sobh MA, et al. Clinical course and outcome of pregnancies recipients of renal allografts. Transplant Proc. 1997;29(7):2787-9.

14. O’ Donnell D, Sevitz H, Seggie JL, Meyers AM, Botha JR, Myburgh JA. Pregnancy after renal transplantation. Aust N Z J Med. 1985;15(3):320-5.

15. O' Connell PJ, Caterson RJ, Stewart JH, Mahony $\mathrm{JF}$. Problems associated with pregnancy in renal allograft recipients. Int $\mathrm{J}$ Artif Organs. 1989;12(3):147-52.

16. Tan PK, Tan AS, Tan HK, Vathsala A, Tay SK. Pregnancy after renal transplantation: experience in Singapore General Hospital. Ann Acad Med Singapore. 2002;31(3):285-9.

17. Al Shohaib S. Erythropoietin therapy in a pregnant post-renal transplant patient. Nephron. 1999;81(1):81-3.

18. First MR, Combs CA, Weiskittel P, Miodovnik M. Lack of effect of pregnancy on renal allograft survival or function. Transplantation. 1995;59(4):472-6.

19. Toma H, Tanabe K, Tokumoto T, Kobayashi C, Yagisawa T. Pregnancy in women receiving renal dialysis or transplantation in Japan: a nationwide survey. Nephrol Dial Transplant. 1999;14(6):1511-6.

20. Crowe AV, Rustom R, Gradden C, Sells RA, Bakran A, Bone JM, et al. Pregnancy does not adversely affect renal transplant function. QJM. 1999;92(11):631-5.

21. Pestana JOM, Ramos OL, Ajzen H. Guia clínico para o transplante renal. J Bras Nefrol. 1992;14(2):66-86.

22.Dekker G, Sibai B. Primary, secondary and tertiary prevention of pre-eclampsia. Lancet. 2001;357(9251):20915.

23. Di Loreto P, Chiaramonte S, Dissegna D, Banzato O, Zuccarotto D, Ronco C. Pregnancy after kidney transplantation. Case load of the Transplantation Center of Vicenza. G Ital Nefrol. 2005;22 Supp1 31:S153-5.

24. Muirhead N, Sabharwal AR, Rieder MJ, Lazarovits AI, Hollomby DJ. The outcome of pregnancy following renal transplantation: the experience of a single center. Transplantation. 1992;54(3):429-32.

25.Jones DC, Hayslett JP. Outcome of pregnancy in women with moderate or severe renal insufficiency. N Engl J Med. 1996;335(4):226-32.

26. Cunningham FG, Gant NF, Leveno KJ, Gilstrap III LC, Hauth JC, Wenstrom KD. Williams obstetrics. 21st ed. New York: McGraw-Hill; 2001. p. 1251-71. 